\title{
T-Lymphocyte and Natural Killer Cell
}

National Cancer Institute

\section{Source}

National Cancer Institute. T-Lymphocyte and Natural Killer Cell. NCI Thesaurus. Code C39567.

Group of lymphocytes. A T-lymphocyte is a white blood cell differentiated in the thymus that possesses highly specific cell-surface antigen receptors. A natural killer cell resembles a T-lymphocyte, but it does not express markers of either T or B cell lineage. Its cytotoxic activity is not antibody dependent. 